\title{
DisCo: Middleware for Securely Deploying Decomposable Services in Partly Trusted Environments
}

\author{
Eric Freudenthal, Edward Keenan, and Vijay Karamcheti \\ Department of Computer Science \\ Courant Institute of Mathematical Sciences \\ New York University \\ $\{$ freudent,woodiek, vijayk\}@cs.nyu.edu
}

\begin{abstract}
The DisCo middleware infrastructure is designed to facilitate the construction of decomposable network services that can be deployed into an environment characterized by dynamic network connectivity properties and unstable trust relationships that span multiple administrative domains. Consumers of these services must be able to discover, securely acquire the code for, and install service components over the network with only minimal a priori knowledge of their locations. Once installed, these components must be able to interoperate securely and reliably across the network.

Solutions exist that do address certain of the challenges posed by such an environment, but the application developer is required to integrate and explicitly manage the use of such solutions. DisCo is an umbrella infrastructure, comprised of a set of independent but highly interoperable core components and abstractions, intended to provide programmers with a more complete solution to the problem of writing network services that can cope with change.
\end{abstract}

\section{Introduction}

Increasingly, distributed applications are being called upon to execute in dynamic network environments spanning multiple administrative domains, and in situations where the principals involved are subject to changing trust relationships. This trend is a consequence of many factors, including user mobility and the growing popularity of federated applications similar to those advocated by the web services standardization efforts. For example, a mobile user may need to interact with services in various network domains (such as airports and hotels) without a priori knowledge of whether or not a particular service is available in a domain, what its characteristics are, and more importantly without any prior relationship between the user and the particular domain. Even if such a relationship can somehow be established (e.g., through the use of certificates), ideally one would like to ensure either that the relationship stays valid through the duration of the interaction or better yet, that the nature of the interaction continually evolves to reflect the current relationship.

Although several systems have been proposed and are being used to cope with the underlying issues highlighted by the above example — service discovery, remote interface management, security and trust management—-these systems suffer from two shortcomings. First, most existing solutions generally assume a static view of trust and resource availability and therefore suffer from time-to-check-to-time-to-use (TOCTTOU) [8] vulnerabilities. To take an example, secure communication abstractions such as SSL/TLS do not address the challenge of monitoring of liveness and authorization properties once a connection has been established, leaving it up to the application to both verify that this is the case and to take appropriate action if it is not. Second, and perhaps, more important from the perspective of this paper, is that systems addressing the above concerns are typically structured as standalone entities. The consequence is that application writers need to take responsibility for the integration effort, managing the application lifetime by detecting and reacting to various kinds of changes. Additionally complicating this issue is the fact that little guidance is provided 
for how such integration can be achieved. To take an example, the Jini discovery service [2], on its own offers little support for security, leaving it upto the application writer to incorporate whatever support is deemed appropriate.

In this paper, we describe the DisCo middleware, which has been designed to address the above shortcomings. DisCo targets a component-based model of applications, and consists of a small set of reusable and interoperable abstractions that support (1) locality-aware discovery; (2) decentralized authorization and access-control; and (3) authorization- and liveness-aware inter-component communication. Together with a minimal run-time system capable of on-demand component installation within a container execution environment with appropriate rights, and a design pattern that relies on indirection objects, the DisCo middleware makes it convenient to build and deploy componentbased distributed applications in network environments with dynamically changing trust relationships. DisCo takes over responsibility for tracking and detecting authorization and liveness changes, requiring programmer input only for application-specific handling of a change event. The latter can, in principle, be abstracted into a set of reusable libraries, and is simplified because of DisCo support for component lifecycle management. Although DisCo has been developed as a self-contained system (on top of Java/RMI), the abstractions underlying DisCo, which allow distributed applications to cope with changes in authorization and connectivity, are also likely to be of utility in other componentbased distributed application frameworks such as Object Management Group's CORBA, or Sun Microsystems' J2EE.

The DisCo middleware continues to be a work in progress, but has been under development now for over two years. The design of some of the underlying abstractions have been described in detail elsewhere [6,7], but not as part of a larger system. Our goal in this paper is to describe the overall DisCo application model, the set of abstractions we believe are necessary to support this model conveniently, and the lessons we have learned from developing applications on top of this middleware.

The rest of the paper is organized as follows. In Section 2, we overview the DisCo application and execution models and present a motivating example. Section 3 presents the details of the core abstractions and the DisCo runtime system components. Section 4 reports on our application experience using three applications. Section 5 evaluates the performance costs of DisCo abstractions. We discuss related work in Section 6 and conclude in Section 7.

\section{DisCo Overview}

We first describe the application and execution model supported by the DisCo middleware and then introduce a motivating example.

\subsection{Application Model}

DisCo applications are assemblages of software components. Components can be instantiated at run-time, with instantiated components possessing a unique identity, expressed using a public key. Communication between components takes place via inter-component connections, which are established as required by the application. Depending on the application, components can either implement a portion of the application's functionality, or alternately serve to enhance interactions with another component. Examples in the latter category include components that act as proxies or caches for other components.

DisCo applications are intended to be deployed in environments where trust relationships between principals may be subject to change. This has two implications for the application model.

First, the deployment of a component on a particular node, or its connection to another component are themselves relationships that may need to be revisited as the application execution proceeds. We formally refer to these relationships as coalitions, with the involved entities (components, node execution environments, users, etc.) being members of this coalition. Members of a coalition may not share complete trust and additionally, these trust relationships may change over time (e.g., a host's security may have been determined to be compromised). As we describe below, the DisCo execution model provides support for three kinds of such coalition relationships: (i) between a user and a host, (ii) between an application component and the node execution environment where it executes, and (iii) between a pair of application components. It is these coalition relationships that may change, as part of application adaptation to changes in resource availability, authorization, or usage.

Second, the DisCo application model needs to deal with situations where an application component may get "left behind" on a host that was initially trusted but which no longer is. To ensure that overall application integrity does not get compromised by this unreachable component, we need to make some assumptions about the application structure. In particular, the DisCo application model requires that application components belong to one of the following two classes: 


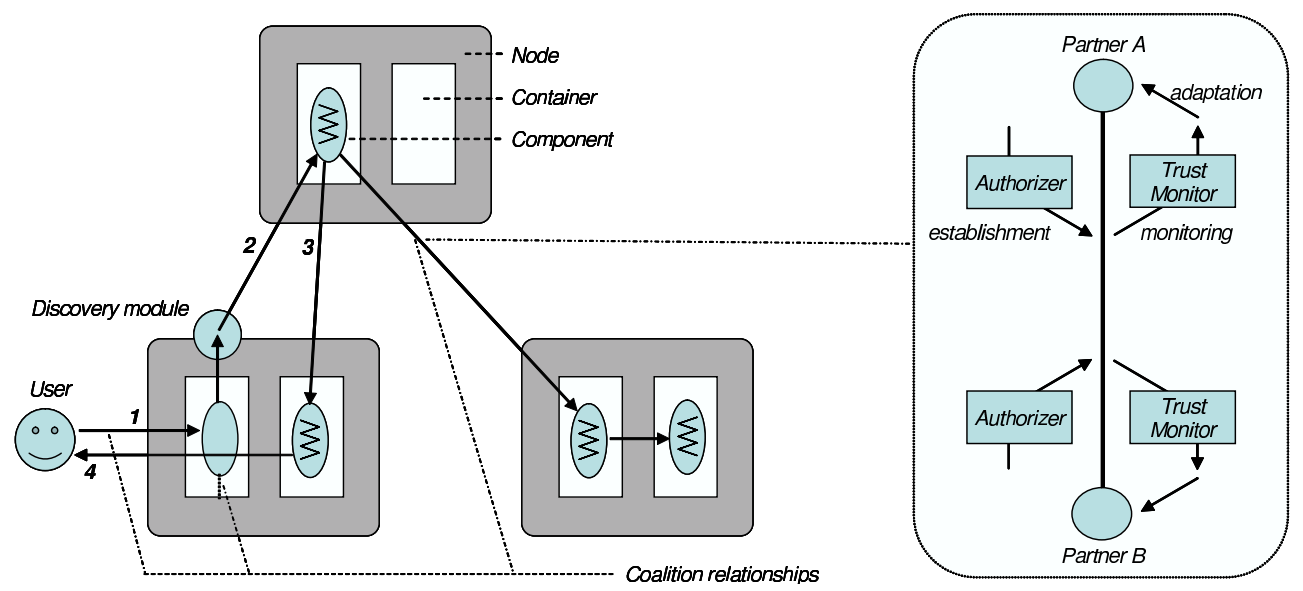

Figure 1. DisCo execution model showing users, nodes, containers, components, and coalition relationships. The figure on the right shows the elements involved in a coalition relationship between two partners $A$ and $B$, for establishing and monitoring it, and adapting to any changes. See the text for an explanation of the labeled arcs.

- The first class includes restricted components with limited functionality that may be abandoned on a host that can no longer be trusted. Examples include proxy implementations that forward sensitive queries to a trusted host, providing useful solutions to queries without compromising the security of the problem-solving code.

- The second class of applications derives its security from the interactions that flow between the various components. For such applications, the underlying system can cope with unpredictable changes in trust simply by controlling whether or not a particular component is allowed to interact with the other components. Examples of such applications include those whose components generate, process, and forward data streams, such as an information dissemination service. In this case, updates on the data stream can be stopped if a previously trusted host were to become untrusted.

Note that proprietary commercial applications that are increasingly being offered to clients on a pay-per-use or subscription basis, or with an expiration period (e.g., virus checking software), have the same kind of distribution and trust concerns.

\subsection{Execution Model}

The high-level execution model supported by the DisCo middleware is best described as follows (see Figure 1). Users desiring access to application services log onto a host and request that the desired application be started (Step 1). This request is propagated to a deployed application component instance, located using a locality-aware discovery service (Step 2). Requests propagated to component instances result in the installation of one or more components on the requesting host, and possibly additional components on other hosts (Step 3). Instantiated components are connected to other deployed components, with the deployment together realizing the overall application. Note that the downloaded component can range from a simple stateless stub to a stateful proxy. Components run in containers, which are host execution "sandbox" environments with appropriately modulated rights. After installation, the user can receive the desired service by interacting with the application component (Step 4).

As stated above, this execution model involves three kinds of coalition relationships: between the user and the host, between a component and its container, and between a pair of components. The DisCo execution model provides support for (1) authorizing (and thereby setting up) the coalition; (2) continuously monitoring the relationship, specifically its authorization and connectivity characteristics; and (3) adapting to changes in these characteristics.

\section{Establishment of a Coalition Relationship}

DisCo establishes a coalition relationship by matching the authorization requirements of the two potential partners with 
each correspondent's authenticated public-key identity and credentials they present as evidence of their eligibility.

Although the current DisCo implementation relies on a distributed role-based trust-management system called dRBAC [6] (see Section 3), its use is encapsulated within a generic abstraction for continuous authorization that allows the selective adoption of alternate trust management systems. This abstraction defines two universal authorization interfaces: an authorizer, and a trust monitor. Each partner in a potential coalition provides an authorizer that is responsible for initially evaluating credentials and authorizing the coalition's formation. If authorization is granted, the trust monitor is responsible for overseeing the partner's eligibility throughout the relationship's lifetime. A trust monitor provides interfaces to receive credential updates sent by the coalition partner, and to alert the relevant coalition management system about loss of authorization.

\section{Authorization and Connectivity Monitoring}

DisCo continuously monitors the authorization underlying a coalition relationship using the trust monitor interface introduced above. In addition, for coalition relationships involving pairs of application components, DisCo also includes mechanisms for monitoring the transmission time and liveness of inter-host communication channels through a tamper-resistant protocol of handshake messages. Connectivity requirements for a particular relationship can be specified by either partner in a coalition. Coalition partners are notified when connectivity requirements are not met.

\section{Adaptation to Loss of Authorization or Connectivity}

The termination of a coalition relationship can occur voluntarily or instead be forced by the loss of an authorizing relationship or the failure of a communication channel (detected as a violation of desired connectivity characteristics). In most cases, partners are notified and provided the opportunity to adjust their requirements or form other coalitions. The only case where a breakdown of the coalition relationship is not communicated is when a component loses all of its own execution privileges in its container since notification requires execution. Note however, that components that have a relationship with this aborted component will detect its absence and therefore themselves be able to adapt.

Potential adaptation responses include the formation of coalitions with alternate components and the installation of new compnents into available containers. DisCo provides mechanisms to inspect pending communication and method calls to the coalition partner, and to choose their disposition. Available responses include transparently replaying these calls to new partner(s), modifying the calls in an application-specific fashion, or aborting them.

\subsection{Motivating Example}

Let's examine how a fictional component-based Internet travel service, TravelAnywhere, is accessed by users in corporate travel departments. As with most Internet travel services, TravelAnywhere's primary services include a query subsystem for searching and choosing among available flights, and a booking subsystem that allows users to purchase tickets. These two subsystems, and a user interface front-end component are packaged as separate DisCo components. These components have the following security and integrity characteristics:

- The query subsystem is compute-intensive, and given TravelAnywhere's interest in providing fast responses to its user queries, can benefit from deployment in proximity to users of the service. However, any corruption in the data used to generate query responses (which is cached in the component) is not desirable. To tradeoff between these two issues, TravelAnywhere permits the query component for a particular corporate entity $C$, to only be deployed on hosts trusted by $C$.

- The booking subsystem is responsible for both financial and reservation transactions. Incorrect client authorization or accounting can result in substantial loss of revenue or financial liability. Therefore, TravelAnywhere is only willing to have the booking component execute on hosts it itself authorizes.

- The user interface component prefers to be located in proximity to the appropriate query subsystem, so as to provide fast responses to user queries.

The DisCo middleware facilitates the deployment of this application in a network comprising a variety of potential execution containers. For example, TravelAnywhere may have installed dedicated systems that they directly authorize. In addition, other vendors may contract with TravelAnywhere to provide hosting containers with acceptable security properties. In both cases, TravelAnywhere issues credentials authorizing their hosts and indirectly, the hosts authorized by the contracted hosting services to execute their components. Similarly, the contracted hosting services provide credentials authorizing TravelAnywhere components to execute in their containers and have access to appropriate 
system resources. Additionally, the network includes hosts that are trusted by the different corporations that use TravelAnywhere's services.

The challenge of deploying the application in this environment stems from having to ensure that the integrity constraints of the components are preserved despite changes in trust relationships, e.g., resulting from an expiration of the contract between a vendor and TravelAnywhere and between a corporation and its trusted sites. To illustrate how the DisCo middleware copes with this challenge, consider a user Sally, an employee of Acme Industrial, who wishes to use TravelAnywhere's service on one of Acme's workstations $W$ :

1. Sally logs on to $W$, which essentially entails her forming a coalition with a set of components $L$ responsible for authorizing logins on and negotiating user coalitions with programs in order that they are able to act on her behalf.

2. Sally requests that the TravelAnywhere application user interface component, $U I$ be started. If $U I$ is not already present on $W$, a provider for $U I$ is first located using the DisCo discovery service, which results in the component getting installed on $W$. Credentials are provided to $U I$ so that it may act on Sally's behalf.

3. $U I$ has a preference for accessing instances of the query component on the local system. Using the DisCo discovery service $U I$ locates a provider of the query component $P$, and requests that it install an instance $Q$ of the routing component on a new container of $W$. Note that TravelAnywhere does not trust $W$, and therefore $Q$ is only authorized by $P$ to service requests from users who trust $W$. $Q$ 's container on $W$ in turn only allows it permissions provided to components authorized by TravelAnywhere as dictated by Acme Industrial.

4. To be able to maintain a consistent view of route availability, $Q$ establishes coalitions with other query components that have appropriately authorized connections to the routing database. $Q$ may also also register itself with the discovery service as a provider of query components authorized for Acme users.

5. $U I$ establishes a coalition with $Q$, and discovers an already installed booking component $B$, with which it also forms a coaltion.

6. Application integrity results from the middleware continuously monitoring the authorizations underlying each of the coalition relations: between Sally and $W$, between the components $U I$ and $Q$ and the host $W$, and between pairs of components $U I-Q, U I-B$, and $Q-Q^{\prime}$ (where Q' refers to other query components in the system). If any of these authorizations no longer hold, the corresponding coalition is terminated, and the application as a whole adapts by having the disconnected components attempt to discover other live providers (with appropriate trust) and establish new coalitions.

In the next section, we describe in additional detail the core components of the DisCo middleware that facilitates the above sequence of actions.

\section{Architecture}

The advantage of DisCo's middleware infrastructure is that it allows application developers to create decomposed network services for use in environments with dynamically changing trust relationships whose components can (1) be securely deployed to remote hosts and installed at runtime and (2) will reliably and securely interoperate with service components installed both locally and on remote hosts. A more detailed list of the features of a network service implemented using the DisCo infrastructure includes the following:

- Service Establishment

- Dynamic service component deployment

- Lazy code distribution

- Secure code distribution

- Local execution of potentially untrusted components is confined to appropriately restricted contexts

- Monitored Authorization and Communication 
- Maintenance of parameterized inter-component connection security and transport properties, including secrecy, integrity, timely delivery, liveness, and timely response to changes in the trust relationship that authorized the connection

- Ability to register a callback that handles in an application-specific manner any failure to meet parameterized requirements of the above security and transport properties

- Adaptation to Environment Changes

- Failover connection to alternative remote components should connectivity or authorization be lost

- An application- and connection-specific level of indirection between connected service components which may be used to implement security filtering, alternative functionality, performance modulation, etc.

- Management of the lifetime of connection objects (cleanup, garbage collection, etc.)

- Aggregation of function calls (broadcast) to connected components of the same type

DisCo is an umbrella infrastructure, comprised of independent libraries, abstractions, and core components whose functionality is combined in order to provide the above features. The infrastructure components can be grouped conceptually into three classes. First is a set of reusable and interoperable abstractions for discovery, decentralized authorization and access control, and authorization- and liveness-aware component communication. These are described below in section 3.1. Second is a design pattern that relies on indirection objects to implement functionality to address coalition-specific concerns. This is described in section 3.2 below. Third is a minimal run-time system capable of on-demand component installation within a container execution environment with appropriate rights, described in section 3.3 below.

\subsection{Reusable Abstractions}

The set of reusable and interoperable abstractions employed by DisCo can be further divided into three conceptual groupings: those used for authorization and access control over disparate trust domains, those used for secure and liveness-aware communication, and those used for locality-aware discovery. A common feature of all three groupings is that they are all designed to indicate or respond to any changes in the environment in a timely manner.

\subsubsection{Decentralized Authorization and Access Control}

dRBAC DisCo requires mechanisms to authorize sustained coalition relationships between entities that may be parts of disjoint trust domains that are unwilling or unable to agree upon a common third party to provide authorization functionality. In addition, while some authorization decisions are binary, others are extended to include multiple permissions at modulated levels. For example, given a credential that grants a service component permission to be installed on a given host, it may be desirable that the credential also explicitly specifies a restricted set of system access rights under which the component would run. To address these needs, we developed dRBAC.

dRBAC is a role-based trust management system for coalition environments. dRBAC credentials, called delegations, express the mapping of an equivalence class of access rights in one trust domain to members of another equivalence class, possibly in another trust domain. Each of these equivalence classes is represented by a dRBAC role. These delegations potentially include attenuation of valued attributes. A a summary of relevant features of dRBAC follows; a more complete description appears in [6].

Each dRBAC delegation is cryptographic ally signed by its issuer. ${ }^{1}$ As with other role-based access control systems, dRBAC delegations may be transitively chained to form proof graphs indirectly authorizing a required class of access rights. A dRBAC credential can be tagged with expiration dates and also may additionally require online validation monitoring from an authorized "home" which is aware of any revocation of the delegation.

Using dRBAC, a trust-sensitive component $C$ can determine if a set of dRBAC credentials $X$ gives some subject $S$ the set of access rights represented by a role $R$ continuously over some duration. To do this, $C$ presents the public identity of $S$, a set of required access rights $R$, and the credentials $X$ to a dRBAC implementation. The dRBAC module first authenticates the signatures and establishes monitors on the validity of all credentials in $X$. Authorization is granted if the dRBAC module can construct a graph from valid and authenticated credentials in $X$ that "proves" that $S$ possesses the rights required by $R$. As evidence of the authorization, a proof monitor object $P$ is constructed

\footnotetext{
${ }^{1}$ Additional credentials may be required as evidence of the issuer's authorization to administer the rights proved by the delegation.
} 


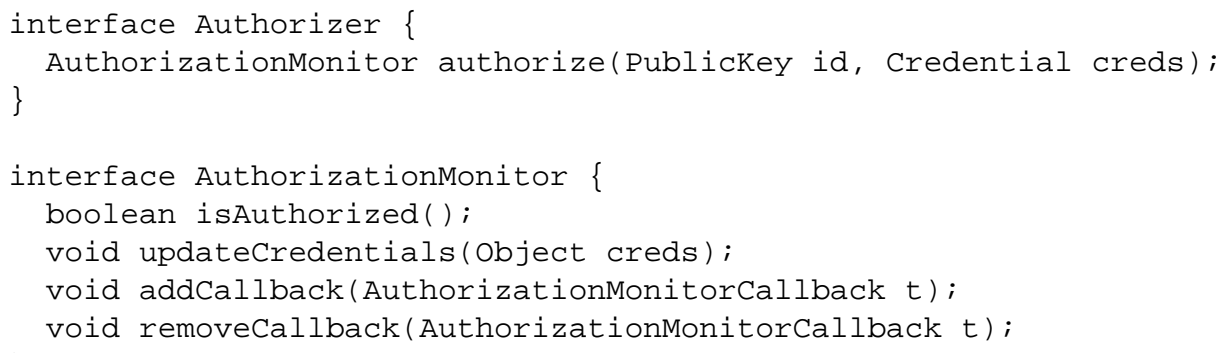

Figure 2. Authorizer and AuthorizationMonitor interfaces

and returned to $C$. $C$ can register a callback with the $P$ so that $C$ can be notified should $S$ lose its rights to $R$. $P$ also provides interfaces to determine the values of attenuated access attributes that are included in the authorizing credential graph. Recall also that the duration of an authorized coalition relationship may exceed the lifetime of the credentials that initially authorized it. In order to avoid loss of authorization, $P$ includes a mechanism for providing updated credentials.

dRBAC credentials are stored in a distributed repository. To assist in collecting dRBAC credentials that authorize a particular partnership, an automated credential discovery mechanism has also been constructed.

Authorizers and Authorization Monitors Trust-sensitive coalition relationships in DisCo are authorized using a generic abstraction called an Authorizer. Authorizer objects are instantiated for each class of potential trust relationships in a DisCo system and evaluate authorization requests that contain only client identity and credentials. If the subject has the required access rights, a DisCo authorizer returns a DisCo Authori zationMonitor that, like a dRBAC proof monitor, contains an interface for installing callbacks and updated credentials. An AuthorizationMonitor provides a mechanism by which an authorization component, such as dRBAC, can diligently watch the status of the authorized trust relationship and provide timely callbacks should that status change.

An authorization monitor's isAuthorized method facilitates the traditional authorization-per-transaction mode of operation. We note that this model of operation can be quite expensive for conventional passive authorizers that only execute in response to a request. In contrast, isAuthorized is is very inexpensive and has low latency when implemented for active authorization monitors such as ones that utilize dRBAC proof monitors since (1) isAuthorized only needs to check a boolean variable to validate authorization and (2) the computation required to maintain authorization is performed asynchronously from queries. In order to be compatible with both active and passive authorization monitors, we suggest that it is best practice for trust-sensitive objects to confirm authorization of each operation by calling isAuthorized.

The DisCo library includes Authorizers and AuthorizationMonitors constructed upon dRBAC. However, the trustmanagement system implemented within an Authorizer and the contents of its corresponding credential objects are opaque to all other DisCo core components. Alternative trust monitors that implement other access control and trust management systems such as X.509 [11] or the $R T_{n}$ [15] systems could be easily constructed and be used seamlessly throughout DisCo. To facilitate the transparent replacement of authorizers, DisCo does not specify the format of credentials, which it treats as opaque objects.

Figure 2 shows the signatures of DisCo classes Authorizer and AuthorizationMonitor. A dRBAC Authorizer for DisCo contains the dRBAC role and attenuation attributes that authorized subjects must possess. The DisCo library contains a convenient factory object to generate appropriate dRBAC Authorizers. dRBAC ProofMonitors extend the AuthorizationMonitor interface.

\subsubsection{Authorization- and Liveness-Aware Inter-Component Communication}

Switchboard The Switchboard [7] library provides a trusted substrate for secure and authorized inter-component communication. Switchboard coalition partnerships are secure, authenticated, continuously authorized connections whose communication channels are monitored when the communication channel uses an external network. 


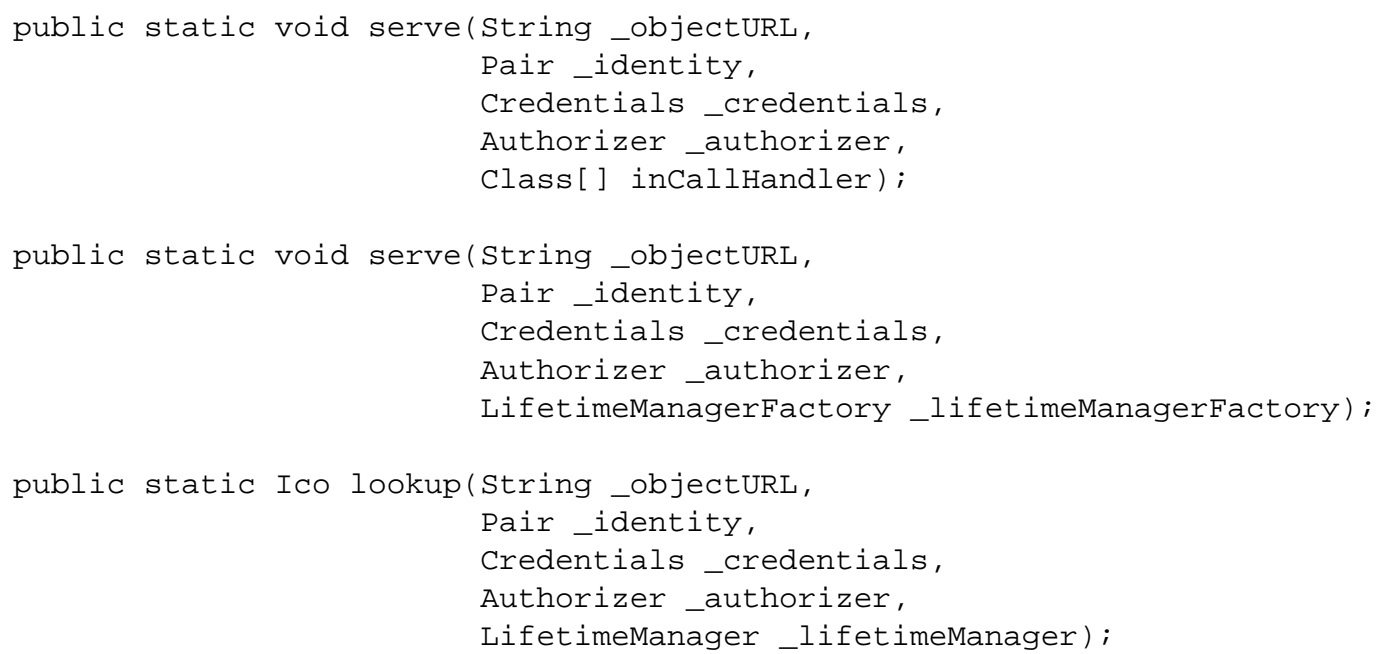

Figure 3. API for serving and looking up remote interfaces over SwitchboardRPC

Prior to forming a Switchboard coalition, both partners provide their authorization suites-PKI identities (including private keys for authentication), credentials to be supplied to the partner, and Authorizers for evaluating partners' eligibility. The Authorizers generate AuthorizationMonitors which will inform the partner when the trust relationship changes.

When a Switchboard connection spans multiple hosts, a cipher is established using a key-exchange protocol, and connectivity is monitored using replay-resistant heartbeats that indicate liveness and round-trip latency. For inter-host Switchboard connections, liveness and the time to deliver messages is monitored so that partners can be informed when a parameterized limit is violated.

A LifetimeManager object provides the logic to handle connection events such as changes in trust or violation of connectivity thresholds, and it provides callback interfaces to be informed when any of these events occur. A Switchboard partner provides either a LiftetimeMonitor for a single connection or a factory with which Switchboard may generate one for each connection.

Three variants of Switchboard are available:

- SwitchboardObj: a bi-directional object transport

- SwitchboardStream: a bi-directional stream transport

- SwitchboardRPC: a bi-directional RPC mechanism

Switchboard provides stream, object, and two-way procedure-call (RPC) interfaces, and performs short-circuit communication when partners reside on the same host. A previous version of SwitchboardStream that provides secure and monitored transport is described in [7].

The interface for RPC over Switchboard is described in Figure 3. The two serve methods differ in the complexity of connection lifetime management. Both versions take a local server URL at which calls are accepted the standard "authorization suite" seen frequently in DisCo: the public and private key identity of the server, the server's credentials, and an authorizer implementation that will authorize connections. The difference in the two versions is that the first simply accepts the Class array of interfaces that SwitchboardRPC will proxy on the client end. Switchboard will generate a basic LifetimeManager for each connection that implements a simple binary protocol in which the connection with the client is broken should any connection or trust alarms be raised. The second version accepts a LifetimeManagerFactory which generates LifetimeManagers that may map client credentials to arbitrary variant implementations of the served interface and may provide application-specific logic to handle connection events.

The lookup method takes the server URL, the standard authorization suite, and a LifetimeManager to handle connection events. It returns an ICO object that provides the requester with an out-call proxy to the desired remote interface and encapsulates all the objects necessary to manage the connection. ICOs are described in greater detail in section 3.2 . 


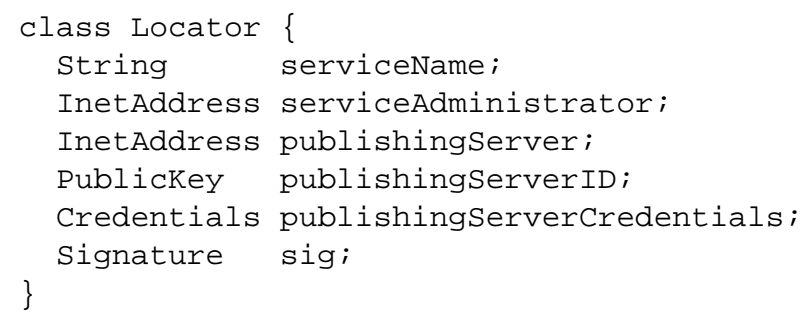

Figure 4. Locator and ServiceDescriptor interfaces

\begin{tabular}{|l|l|}
\hline Service Advertisement: & $\begin{array}{l}\text { void advertise (Locator loc); } \\
\text { void unadvertise (Locator loc); }\end{array}$ \\
\hline Service Publishing: & $\begin{array}{l}\text { void publish (Locator loc); } \\
\text { void unpublish (Locator loc); }\end{array}$ \\
\hline Service Discovery: & $\begin{array}{c}\text { Locator [ find (ServiceDescriptor s, } \\
\text { Authorizer auth); }\end{array}$ \\
\hline
\end{tabular}

Figure 5. Interfaces for DisCo's Discovery module

\subsubsection{Locality-Aware Discovery}

Locator and ServiceDescriptor The Locator is a signed, verifiable resource descriptor token used by DisCo to uniquely identify network services. A Locator contains sufficient data to initiate a Switchboard connection to the resource described in the Locator. As shown in Figure 4, the members of Locator include the following:

- serviceName and serviceAdministrator uniquely identify a given service by its human-readable name and the Internet domain that originally authored it.

- publishingServer is the IP address, port, and server path at which the service is bound and published.

- publishingServerID is the Public Key identity of the server publishing the service. Verification of the signature sig using the provided public key will prove that the creator of the Locator is in possession of the corresponding private key.

- publishingServerCredentials provide the credentials of the server (in whatever underlying credential model is in use: X509, dRBAC, etc.) so that the consumer of the Locator may determine that the server has required security properties before further interaction.

- sig digital signature of the above fields.

A ServiceDescriptor contains the unique identifier fields of a Locator.

Discovery DisCo's Discovery library gathers Locators for a requested service. Caching extra Locators provides a failover mechanism, as it allows DisCo services to reconnect to a different, valid service provider in the event that the current service provider becomes unreachable or unsuitable. Discovery provides three mechanisms as shown in Figure 5. The Service Advertisement interface controls broadcast of Locators, describing the service to be advertised. The Service Publishing interface allows a service to tell the the local Discovery module to passively wait for and respond to requests that match the ServiceDescriptor elements of a provided Locator (as opposed to actively broadcasting them as in the service advertisement interface). Finally, the Service Discovery interface sends out a request for Locators 
that offer the service specified in a ServiceDescriptor. The find method also takes an Authorizer that contains the credentials required of any returned Locators and the mechanism for evaluating them.

\subsection{DisCo Indirection Design Pattern}

In order to flexibly respond to unstable trust relationships, DisCo relies on a design pattern in which all interactions between a component and any other connecting component pass through an indirection object. This pattern allows the application programmer to provide a modular implementation of the served interface specific to the application and trust relationship of the connection.

The AuthorizationMonitor on the server side of a given Switchboard connection will contain the credentials of the component that requested the connection. The server component may examine these credentials to determine what interface instance to return in response to the request, considering any number of application-specific criteria: the need for stateful or stateless connections, heavy or lightweight implementations for VIP or basic-level customers, connection quality, etc. In this way, a service may provide an instance of service functionality for each connection, for each class of connection, for all connections, or any combination thereof.

Indirect Component Object (ICO) The indirection model is embodied in the ICO. An ICO manages the overhead of a single SwitchboardRPC partnership, aggregating all of the necessary objects and facilitating interoperation between them. An ICO contains the following components:

- An In-Call Handler, the remote-procedure-call interface provided to the partner. This is a reference to the actual server-side implementation of the served interface. This implementation may be one of many variants and instances that could be provided.

- An Out-Call Proxy, the stub provided to partners. Out-call proxies have the same signatures as their corresponding in-call handlers.

- A Switchboard Connection Manager object: the transport between ICOs. The connection manager also provides interfaces to the partnership's liveness and authorization monitors.

- A Lifetime Manager is provided by the server application component. It is notified of connectivity and authorization events by the connection manager, and it contains logic to handle these events. This logic will govern failover response, modulation of service quality, etc.

ICOContainer and Hydrant An ICOContainer is a collection of ICOs. Upon creation, each ICO is added to the ICOContainer so that when a connection is ended, the ICO is able to inform the ICOContainer that it may be cleaned up and disposed of gracefully.

A Hydrant is an ICOContainer in which are kept ICOs to equivalent service components. The Hydrant will manage aggregate apply calls to each ICO in the Hydrant. Figure 6 shows a service installation $P$ that is providing implementations of the interface ServiceX over SwitchboardRPC. Switchboard on P's host creates an ICO when a distributed component $C$, a consumer of ServiceX, attempts to initiate a SwitchboardRPC connection and call methods on a ServiceX implementation. As it creates the new ICO, Switchboard gets a new LifetimeManager $L$ from the LifetimeManagerFactory provided by $P$ when it began to serve ServiceX over SwitchboardRPC. $L$ examines the credentials that $C$ provided to authorize its Switchboard connection to $P$, and $L$ sets the ICO's In-Call Handler to a reference to $X_{k}$, a ServiceX implementation appropriate to what $L$ finds in $C$ 's credentials. $X_{k}$ may implement a version of each of the methods defined in ServiceX, or it may implement a variant of some of the ServiceX methods and trampoline others directly to another implementation of ServiceX, or it may be a ServiceX root server (should $C$ enjoy $P$ 's full and unshakable trust). Switchboard gives the ICO a reference to the ConnectionManager - the object handle on the Switchboard partnership between $P$ and $C$. Switchboard then gives the LifetimeManager a reference to the ConnectionManager, and the LifetimeManager exchanges callbacks with the ConnectionManager's Authorizationand ConnectionMonitors so that they may inform the LifetimeManager of connection events (changes in trust and connectivity), which it can then handle in an application-specific manner: telling $X_{k}$ to handle calls differently, telling the ConnectionManager to close or alter the Switchboard connection, etc. Switchboard then returns to $C$ an ICO of its own. From this ICO, $C$ may retrieve the Out-Call Proxy that is connected to the In-Call Handler $X_{k}$ over SwitchboardRPC. $C$ is thus able to make calls on an implementation of ServiceX that is appropriate to the connection and level of trust it shares with $P$. All necessary credentials and AuthorizationMonitors are in place so that should the desire or 


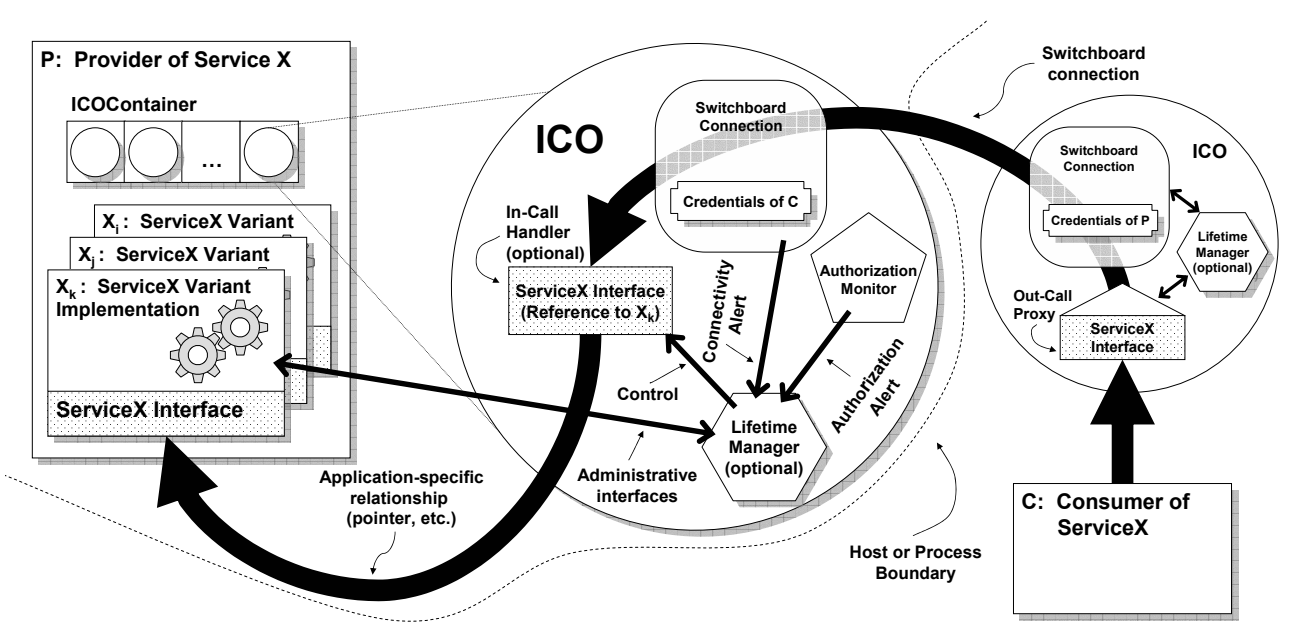

Figure 6. DisCo objects used in a SwitchboardRPC connection through an ICO

need arise, $C$ may use its ICO to pass Switchboard an In-Call Handler and a LifetimeManager that SwitchboardRPC can then use to provide $P$ with an Out-Call Proxy of some interface served by $C$. If $P$ has received Out-Call Proxies of the same type to all its connected remote component consumers of ServiceX (including $C$ ), it could then use the ICOContainer that holds the ICOs as a broadcast mechanism, applying aggregate method calls to all connected components.

\subsection{Runtime System}

The elements that make up the DisCo component runtime system are designed to be responsive to changes in the environment. Objects may need to be instantiated on hosts to provide services that were not available when the host was initially configured. These objects need to be provided appropriate access rights that may need to be modulated if authorization changes. The facilities that DisCo provides to free the application programmer from these concerns are described here.

Under DisCo, an object's access to communication and system resources are mediated through three mechanisms: (1) Java execution environments prevent "pointer forging" and thereby provide insulation against unauthorized tampering with private components; (2) each object's direct access to system resources is limited by Java's security manager infrastructure, which is exploited to create secure execution "sandbox" containers; and (3) Switchboard as a secure and flexible substrate for inter-object communication.

Execution Containers and ContainerSecurityAuthorizers All objects within DisCo execute within restricted sandboxes, called Containers, which have controlled access to system resources. Java 2's security model includes a "secure class loader" specification that DisCo exploits to both implement execution containers with appropriate access restrictions and to securely obtain code for objects not provided when the host was initially deployed.

Java's secure class loader interface provides a mechanism for objects created on remote hosts to obtain execution binaries - class files - and to establish object security contexts. Using this mechanism, the system administrator specifies a security policy that includes the authorization of a set of secure class loaders. Objects can be transferred between hosts in a serialized format that includes an annotation of the code source. If a serialized object's code source is honored by an authorized secure class loader, then the class loader both provides the binary class file and specifies the object's access rights as expressed by a security manager object.

The DisCo secure class loader uses a Switchboard partnership to securely transfer a serialized object's class file. Rather than referencing a specific agent authorized to provide code files, DisCo secure class loaders utilize the DisCo locality-aware discovery service to obtain code from nearby hosts.

DisCo hosts require protection against rogue objects loaded from external sources. ContainerSecurityAuthorizers provide a bridge between a trust management systems (X509, dRBAC, etc.) and administrative security policy. As with Authorizers, ContainerSecurityAuthorizers are factories for ContainerSecurityMonitors. ContainerSecurityMonitors 
function as binary Authorizers to permit the downloading of class files and object instantiation. They also provide a permission vector that represents container access privileges to system resources.

Secure class loaders (which are trusted by the host) create a Container based on the ContainerSecurityMonitor's permission vector, limiting the abilities of the service component code executing inside it. The ContainerSecurityMonitor modulates the permissions of the Container accordingly if the credentials used to create the Container change.

ContainerSecurityMonitors provide natural insertion points for usage accounting since they have privileged access to the container control structures.

Privileged Proxies An object given minimal direct access to system resources may still be granted indirect access through privileged proxies accessible via Switchboard. For example, access to a user's files may not be permitted to objects loaded through a secure codebase. If a user wants to empower such an object $O$ to access the user's files, the user can provide credentials to $O$ permitting it indirect access via Switchboard to a privileged filesystem proxy $P$ authorized to access the user's files. In this fashion, $O$ 's identity, combined with its credentials, function in a manner similar to capabilities.

Component Installation and the DisCo Activator SwitchboardRPC, combined with the DisCo secure class loader, provides a mechanism to securely deploy objects in order to provide services that execute on coalition systems. These objects can be include functionality and credentials that permit them to provide services to both local and remote entities via Switchboard partnerships. In order to allow these components to be strategically deployed onto cooperative hosts, DisCo provides a generic component deployment subsystem called the Activator.

Activators are privileged proxies whose role is to deploy objects on coalition hosts as requested by coalition deployment managers. Following the DisCo design pattern, a deployment dispatcher $D$ requesting the deployment of an object $O$ on to host $H$ requests the formation of a SwitchboardRPC partnership with $H$ 's Activator $A$. This partnership is formed only if $D$ has authorization required to request activation on $H$, as is specified by $A$ 's authorization suite (the PKI identity and credentials of $A$ ).

If the partnership is formed, $D$ provides a locator $L$ for an activatable interface $I$ that provides $O$ via the method getcomponent. $D$ also provides credentials $C_{D}$ authorizing $H$ to download $O$ and credentials $C_{U}$ authorizing $O$ to be unmarshalled and provided execution rights on $H$. $A$ then attempts to establish a SwitchboardRPC connection with $L$ using an authorization suite including $H$ 's identity, $C$, and the host's security policy for activating objects. If this second partnership is formed, $I$ is provided to $A$, and its get Component method is called. $O$ is then returned, its code downloaded (if necessary), and instantiated on $H$ as permitted by $C_{U}$. Finally, $I$ is provided to $O$ in order to facilitate communication with its progenitor.

\subsection{Use of Core Components to Honor DisCo Guarantees}

It is through the use of these constituent core components and abstractions that DisCo is able to honor the security and performance guarantees outlined at the beginning of this section. Figure 7 shows how libraries and abstractions are used in concert and the features that result from these aggregations.

\section{Sample Applications Implemented as DisCo Components}

To illustrate DisCo's utility as an infrastructure for secure deployment of decomposable applications in dynamic partly-trusted network environments, we describe the design of three applications below. Implementation of two of these applications-video distribution and "battleship" game-is complete, while that of the last one - secure mail -is in progress. The secure mail application leverages the efforts of another member of our group, Anca Ivan, who developed the application to investigate automatic deployment approaches for component-based applications [12].

We start off by describing the requirements for each of the applications and their realization as DisCo components, and then discuss the benefits provided by different DisCo abstractions.

\subsection{Application Characteristics}

\section{Video Distribution}

The application consisted of delivering an encrypted video broadcast stream to a set of clients. The security requirements of the application stemmed from the fact that only clients with appropriate credentials should be allowed to receive the stream, and only for as long as these credentials remained valid. 


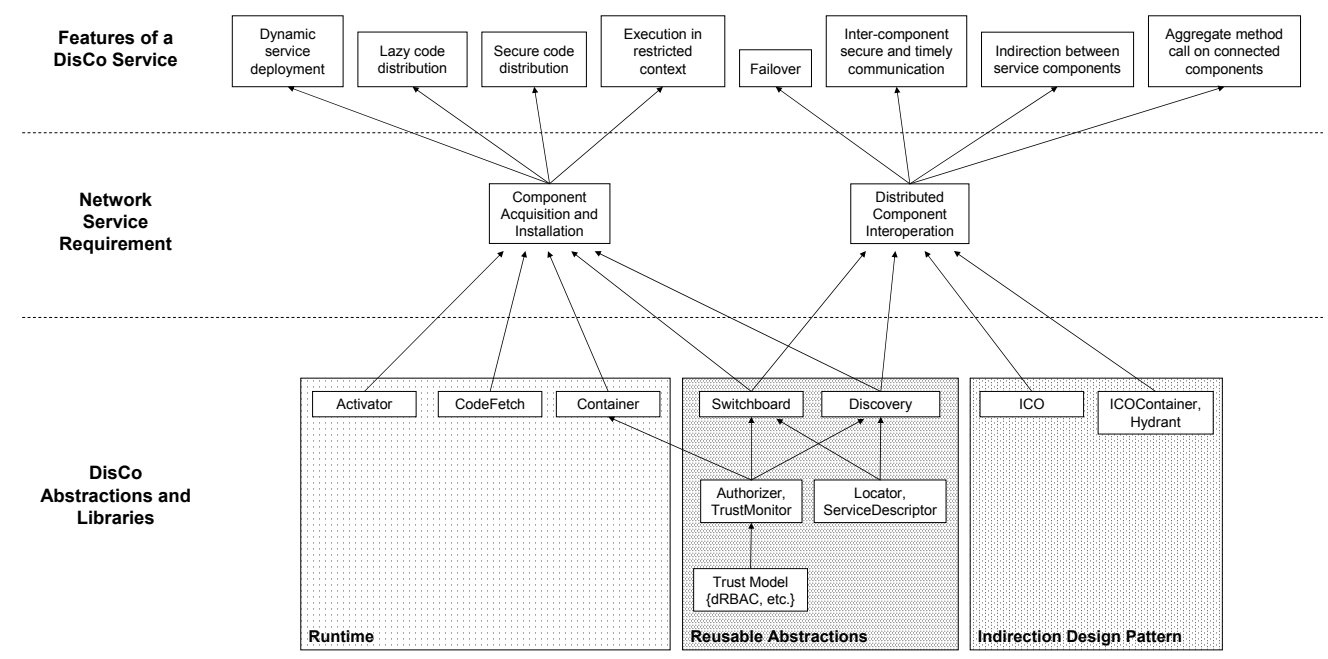

Figure 7. DisCo constituent libraries combining to honor service guarantees

This application was realized in terms of two broadcast networks: one for distributing the encrypted stream (built using the Java Media Framework), and the other for handling key distribution. We assumed the availability of a rekeyable cipher, simulated in our application, which would be rekeyed whenever a client or key distribution agent lost authorization. The application involved three kinds of components: the stream source (including the virtual rekeying logic), a key distribution component, and a stream viewer client, the last two of which could be instantiated in a demand-driven fashion. A user would log onto a host, start the viewer, which would connect to the key distribution component on the local host, which itself would be dynamically instantiated as required. The key distribution component would provide the current decryption keys (that it received from an upstream component) to the viewer. These keys would become useless if the cipher changed, requiring propagation of the updated keys to the viewers.

The sources of implementation complexity for this application include: (1) authorization of clients that may be anonymous w.r.t. the stream source; (2) demand-driven installation of stream-handling components on hosts; (3) enforcement of the security properties, namely that rekeying updates be propagated to authorized users, and that unauthorized users be denied access; and (4) coping with loss of host authorization that may leave a downstream key distribution agent disconnected from the distribution network.

\section{2. "Battleship" Game}

The application consisted of a "single arena" multiplayer game across network nodes, whose structure closely follows the video distribution application. This "war game" provided users with a reconaissance display whose quality was degraded for players and systems with lower trust. Players belonged to teams, with each player receiving information only about his or her team. Player actions (dropping of "bombs" on "targets" belong to other teams) were registered on a master view, resulting in appropriate updates to the individual team views.

As with the video distribution application, this application was also realized as a broadcast distribution tree from a master data feed. Again, there were three kinds of components: a game master component, a distribution component capable of attenuating data quality, and a viewer (built using Java Swing). The latter two components could be dynamically instantiated. Security requirements of the application included restricting whether or not a distribution component could see feeds belonging to multiple teams and attenuating the data sent onto viewers according to the trust level of the user and the host. A performance requirement for the application was that a player not see significant disruptions in feed availability. To support this, the viewer and distribution components were designed to eagerly attach to multiple sources, allowing rapid failover if the primary feed was disrupted for whatever reason.

The implementation complexity for this application arises because of the need to: (1) resolve the required attenuation for data feeds in accordance with credentials of users and hosts; (2) realize this attenuation behavior for a dynamic number of downstream connections; (3) handle loss of authorization of a user or the host where a distribution component is executing. 
3. Secure Mail This application provides a component-based realization of a mail service that is automatically deployed in a dynamic network spanning multiple trust domains. This secure mail service is part of a related research effort (see [12]) that is examining strategies for deployment planning, which satisfy both security and performance constraints.

The hosts and network resources available for this application provide varied levels of performance and security that may vary over time. The component-based structure of the mail service enables component deployments customized to the properties of the network. In particular, the application consists of server, cache, and client components, each supporting multiple realizations. Additional cipher components help realize a security model where each user and mail message has an associated security level. The latter indicates a need for encryption when a message is being transferred through or stored in insecure components. Novel to this application is the use of a separate planning component: unlike the applications described above that deploy components in a greedy manner, the mail service relies on the planning component to compute optimized deployment configurations based on available security characteristics and resources.

Tne sources of complexity for this application include (1) computing optimized deployments that satisfy security and performance constraints; (2) credentialing and deploying appropriate realizations of components; (3) establishing communication partnerships among components; and (4) validating security properties of configurations and enforcing security relationships as properties change.

\subsection{How DisCo Helps}

All three of the applications benefit from DisCo's mechanisms that facilitate the secure discovery, and dynamic installation of and communication between components. At the high level, DisCo allowed the service components to focus only on service-specific functionality issues, taking over almost all of the responsibility for component instantiation, communication, and maintenance of any required security properties.

Coding effort for the first two applications was dominated by application-specific logic rather than deployment issues. The video distribution key management agents, which implemented a trivial algorithm with aggressive security properties, correspond to only a few dozen lines of code that make extensive use of the DisCo facilities as described in additional detail below. The war game application, built upon the same framework as the key distribution system, contains substantially more code to implement signal degradation, display rendering, and the maintainance of game state. However, no additional communication and authorization mechanisms were required beyond those implemented for the key distribution system.

The code for the secure mail service had a larger deployment component. The planning component currently implements an AI planning mechanism, which corresponds to roughly half of the overall source code for the application. Note however that this planning component is reusable over other applications that can benefit from optimized deployment.

\section{dRBAC and Continuous Authorization}

All three applications utilize dRBAC to represent access control authorization and to adaptively react to its loss. In each case, the trust relationships between components and other components and potential containers are represented as dRBAC credentials. This facilitated the convenient mapping of access control decisions to dRBAC authorization and trust monitoring.

In the video distribution application, the key being distributed has only one valid value at any particular time. Therefore, binary authorization of entities - whose roles are to either act as authorized users or key (re)distribution agents is sufficient. dRBAC'S extended authorization model, including valued attributes, provides the other two applications a convenient mechanism for continuously modulating trust relationships between collaborators.

Each of the coaltion partnerships in these example applications is long-lived and therefore suitable for the continuous authorization monitoring provided by DisCo's authorization subsystems. In each case, the mapping of required access rights to dRBAC representations is compact and conveniently enforced by the trust monitor abstraction.

\section{Discovery and Activation Subsystem}

The first two applications utilize DisCo's locality-aware discovery subsystem to drive greedy deployment algorithms that prefer to obtain services from local providers. In both of these applications, components utilize referrals from local systems, or if necessary, contact a centralized service to request a referral. In contrast, the secure mail service only utilizes DisCo's discovery subsystem to locate and install an agent for the deployment planner. After this agent is installed, it, rather than the discovery subsystem, is responsible for deployment and configuration planning and policy. 
The activator subsystem is utilized by all three applications to securely install components onto trusted hosts. The war game, which installs data dissemination components on hosts of varying security, utilizes dRBAC's extended authorization modes in order to choose components with appropriate security properties to be deployed into different hosts. Similarly, the mail service application chooses appropriate realizations of the cache and client components depending on host and user properties.

DisCo's secure codebase facility permits each of these applications to seamlessly pass objects among partner components without having to push all eligible code to all network hosts. This lazy-evaluation mechanism, which extends Java RMI's technique of dynamically loading code on demand, also enables these applications to easily bootstrap-load code for objects that become components on foreign hosts.

\section{Switchboard}

The Switchboard abstractions were utilized extensively by all three applications, serving as the basis for almost all inter-component communication. The video distribution and mail service applications utilize the SwitchboardRPC interface (note that the broadcast of the video stream itself was done out-of-band using JMF protocols). The war game application utilized SwithboardStream for data feed dissemination and SwitchboardRPC to propagate user upcalls to the master view. In each case, the authorization and connectivity characteristics of these communications are continuously supervised by dRBAC trust monitors.

The primary benefit of the Switchboard abstractions is the decoupling of data transfer between the components from additional code required to verify and enforce security properties.

\section{Indirection Objects}

The ICO abstractions are utilized by all three applications, serving to encapsulate repair code that is responsible for handling loss of authorization or connectivity on a communication channel. In the video distribution application, this code is responsible for discovering and connecting to a different key distribution component if the previous connection becomes unusable. In the war game application, the code switches over to the backup feed, while in the mail service application, this code is responsible for interacting with the planner component and requesting a new deployment.

The "hydrant" abstraction is utilized by two of the applications-battleship game and mail service. In the war game application, this facility permits a data dissemination component to make a single method call to propagate the data downstream: the objects within the hydrant pattern ensure that appropriate attenuation is achieved for each of the (dynamic set of) subscribers. In the mail service application, the hydrant abstraction is being used to isolate coherence management between server and cache components, or two cache components. Similar to the war game application, coherence is a function of the users supported downstream of subscriber component.

\section{DisCo Status and Performance Implications}

DisCo is being implemented as a set of Java libraries, building upon functionality contained in Sun Microsystems' JDK 1.4. The latest snapshots of the DisCo code can be downloaded from our web site: http://www.cs.nyu.edu/pdsg (follow the Software tab).

\subsection{Implementation Status}

Although work on the DisCo project is ongoing, prototype implementations exist of all major DisCo subsystems, with the exception of containers and containerAuthorizationMonitors, which are under construction.

Additionally, the application studies conducted to date rely on a simpler version of the secure code distribution subsystem than that described in Section 3. This version consists of a local secure code proxy CodeFetch and a traditional network file server (Apache http server, etc.), which ensure, using the strategy described below, that the class code is available only to those hosts that have already obtained a serialized object through other means.

All serialized Java objects are annotated with the codebase from which they were originally loaded. The CodeFetch approach to secure code distribution is to use altered codebase URLs that reference the CodeFetch proxy executing on a reserved port on localhost. The altered URL also contains the following:

- The web address of a web server providing an encrypted and signed version of the required code file.

- A decryption key for the class file. 


\begin{tabular}{|c|c|c|c|c|}
\hline \multirow[t]{3}{*}{ Size } & \multicolumn{4}{|c|}{ Transports } \\
\hline & \multicolumn{2}{|c|}{ Switchboard } & \multirow[t]{2}{*}{ Socket } & \multirow[t]{2}{*}{ TLS } \\
\hline & null & Blowfish & & \\
\hline $1 \mathrm{~KB}$ & $3.2 \mathrm{~ms}$ & $3.9 \mathrm{~ms}$ & $0.1 \mathrm{~ms}$ & $0.8 \mathrm{~ms}$ \\
\hline $8 \mathrm{~KB}$ & $25 \mathrm{~ms}$ & $39 \mathrm{~ms}$ & $0.6 \mathrm{~ms}$ & $5 \mathrm{~ms}$ \\
\hline $32 \mathrm{~KB}$ & $99 \mathrm{~ms}$ & $130 \mathrm{~ms}$ & $2.2 \mathrm{~ms}$ & fail(lockup) \\
\hline
\end{tabular}

Table 1. Round-trip latency of byte stream echo for different message sizes.

Thus an unmodified Java system running with a CodeFetch-formatted codebase string will reference the local CodeFetch proxy which can extract the code server web address and decryption key from the codebase. The CodeFetch proxy can then can in turn obtain, decrypt, and seamlessly supply the runtime with the referenced code file.

\subsection{Performance Implications}

The run-time overheads of DisCo applications are primarily dictated by the costs of the Switchboard abstraction, which mediates all inter-component communication within DisCo. Switchboard comprises three APIs (object, stream, and RPC) and two major operating modes (inter- and intra-host). Here, we discuss the performance implications of the SwitchboardStream and SwitchboardRPC abstractions, and present the performance of our initial implementations. We should note that at the present time, our focus has been primarily on ensuring that the abstractions provide the correct functionality with the consequence that the implementations have not been tuned for performance.

\section{SwitchboardStream}

SwitchboardStream implements a reliable, in-order, bi-directional, and secure stream transport with continuous authorization and connectivity monitoring and is used for all inter-host partnerships.

Implementation: Our current implementation of SwitchboardStream utilizes the TLS [5] key-exchange protocol (SSHv2) and a Bouncycastle [22] Blowfish cipher. While it would appear that SwitchboardStream can directly build on a secure communication transport such as TLS, we needed to construct our own transport because TLS does not authenticate a partner's identity if X.509 authorization is not utilized.

The ICO's associated with SwitchboardStream simulate a Java socket API by providing piped streams to correspondents. Two independent threads associated with each ICO copy data between these piped streams and an encrypted socket. All messages include headers with fields to support liveness monitoring. An additional "timestamp monitor" thread is responsible for computing connectivity characteristics, and generating "heartbeat" messages if the line has been idle too long.

Performance: The costs of SwitchboardStream can be classified into three categories: transport latency, authorization monitoring, and connectivity monitoring.

To measure the transport latency, our experiment repeatedly passes a fixed length message between two partners executing on the same host (SwitchboardStream is forced to use its inter-host mode). Table 1 presents measurements for our transport with a null cipher and the Blowfish cipher respectively, and compares it with costs seen by JDK 1.4 socket (unencrypted) and TLS implementations. Our initial implementation of SwitchboardStream has significantly poorer performance than Java's TLS implementation. A large fraction of this cost stems from the need to implement connectivity monitoring (see below) in the context of blocking semantics. The latter has the consequence that all received data must be handed-off from a receiving thread associated with the Switchboard connection to the client thread. This handoff presently involves thread synchronization and double buffering. We are investigating a nonblocking variant of Switchboard that should result in substantially better performance.

Authorization of a Switchboard partnership is monitored by DisCo's "authorizationMonitor" mechanism, which is external to the Switchboard implementation. This mechanism operates asynchronously in the background, with the result that consultation of such active monitors may only require a negligible amount of "online" computation.

Connectivity monitoring, described in Section 3.1.2, requires that ICOs have evidence that their remote partners remain reachable in a timely manner. If the Switchboard connection sees periodic data traffic, then no additional overheads are incurred. In the absence of such traffic, the timestamp monitor thread will generate periodic heartbeat messages, whose overhead is less significant because it does not interfere with data transmission. 


\section{SwitchboardRPC}

SwitchboardRPC mediates most inter-component communication within DisCo. The abstraction provides RPC functionality by building on top of the SwitchboardStream implementation.

Implementation: The SwitchboardRPC implementation relies on an additional layer of ICOs at either end of a SwitchboardStream connection to implement the OutcallProxy and incallHandler functionality described in Section 3.2. The outcallProxy (stub) masquerades as the remote object being referenced. Its role is to marshall the identify of the called method and its arguments and call the appropriate SwitchboardStream function for transmitting the invocation. In our present implementation, outcallProxies are implemented as Java "dynamic proxies". The incallHandler (skeleton) is responsible for unmarshalling the arguments and calling the referenced function and returning the result (either a return value or an exception). SwitchboardRPC presently implements incallHandlers using Java's introspection API. Note that intra-host partnerships are efficiently implemented as subroutine calls between protected interfaces of ICOs, avoiding the need for object serialization, liveness monitoring, and encryption.

Performance: The use of both dynamic proxies and Java introspection renders the SwitchboardRPC implementation at a significant performance disadvantage as compared to Java RMI, the natural non-secure alternative. Experimentation indicates that minimum method calls using SwitchboardRPC on the same host (which measures the costs of going through the outcallProxy and incallHandler) are two hundred times slower than direct method calls. We are presently investigating alternative techniques that automatically generate customized outcall "proxies" and incall "skeletons". Our initial experiments indicate that these techniques will reduce the latency of this indirection by more than an order of magnitude.

\section{Related Work}

DisCo is an integrated framework for constructing distributed security-aware applications on systems administered by multiple administrative authorities. This framework is built as several subsystems implementing authorization, secure communication, dynamic deployment, sandbox configuration, and locality-aware discovery. In this section, we place our work in context by discussing other systems that address similar challenges.

\subsection{Component Deployment Systems}

The Java Enterprise Edition (J2EE)[21] provides a component-based application model that deploys components expressed as "beans" into containers. The J2EE model is designed for server farms within a single trust domain, where a bean deployment specification is manually generated at the time the application is configured. All code is unconditionally trusted, and inter-bean communication is provided using unchecked (trusted) RMI. While J2EE does provide a level of protection between well-behaved program elements, authorization is only performed at the start of a user transaction. Thus, the J2EE framework as currently implemented does not appear to provide appropriate inter-component guarantees required for systems spanning multiple administrative domains with assymmetric trust relationships.

More general are frameworks such as .NET [17] and the emerging Web Services effort, which are developing standards to orchestrate interactions among static components resident across multiple administrative domains. However, web services security-related standards such as WS-Security [1] only provide mechanisms for encoding security credentials, without explicitly specifying policies for enforcing various guarantees. Additionally, these frameworks have not explored how long-lived connections between components ought to be affected by the loss of authorization or a change in connectivity characteristics.

Dynamic component deployment onto secure containers is being explored in some research projects such as Ninja [9] and Ajanta [13]. DisCo shares several of its objectives with such systems. In particular, DisCo's secure code loading facilities provide an alternative to Ajanta's mechanism for authenticated, encrypted communication with the code base. However, the primary difference arises from the fact that most such systems focus on system-wide deployment using a central trust-management authority.

DisCo differs from the above systems in two important ways. First, it supports dynamic deployment in environments where all entities do not trust a single authority. Second, it provides mechanisms to continuously monitor the authorization of credentials and connection characteristics, and make it convenient for applications to adapt as necessary. Thus, DisCo mechanisms, such as those used to provide components with capabilities effective across multiple trust authorities, are complementary to what is offered by the above systems. 


\subsection{Secure and Quality-Assured Transports}

DisCo utilizes Switchboard as its inter-container and inter-host communication abstraction. Switchboard, which builds upon JDK 1.4's cryptographic interfaces, provides novel security attributes including continuous monitoring of connectivity and authorization. These attributes are either not available in current transport-layer alternatives, or are implemented in a non-modular fashion hampering their general use.

SSH [24] and TLS [5] are the most widely available alternatives to Switchboard. The authorization model they provide is not modular, but instead is tied closely to ACLs and X.509 certificates, respectively. Neither provides mechanisms to monitor connectivity.

IPSEC [14] provides a mechaism to securely construct a secure network among sites connected via the public Internet. While IPSEC does not provide liveness guarantees, its security guarantees permit TCP to reliably detect the failure of a connection. As a network-level mechanism, IPSEC is unaware of the protection domain of components who originate packets or listen to ports and therefore is inappropriate for the security needs of containers that are on the same host, but need to be insulated from each other.

Modular research systems such as Cactus [23] and JXTA [18] provide alternate mechanisms that can be utilized to implement or further generalize the abstractions provided by Switchboard. However suitable toolkits for these systems were not available at the time our implementation of Switchboard was developed.

\subsection{Authorization and Access Control}

DisCo provides a mechanism for a trust management subsystem to continuously monitor a coalition partner's authorization. In addition, DisCo relies on authorization subsystems to set access level parameters. Both of these mechanisms are realized using dRBAC, a decentralized role-based access-control system [6].

X.509, and several role-based access-control infrastructures such as SPKI/SDSI [20], PolicyMaker[3], OASIS [10], and the $R T_{n}$ systems of $\mathrm{Li}$ and Winsboro $[16,15]$ are similar to dRBAC in providing trust management mechanisms that allow access privileges to be chained or delegated among multiple trust domains. Also related, but significantly less expressive, is Project JXTA's Poblano [4] trust-management system, which provides a PGP-like web-of-trust model.

DisCo's dRBAC differs from the above systems in two respects. First, it provides valued attributes, permitting convenient and flexible modulation of access classes instead of the alternative of requiring explicit enumeration of all combinations of access parameter values as different roles. ( $R T_{1}$, which was developed at around the same time as dRBAC, has a somewhat related feature as well).

More importantly, dRBAC provides facilities to continuously monitor an entity's authorization. With the notable exception of the OASIS [10] system, the above trust management systems have not addressed this challenge at any level. Moreover, OASIS' abstractions and implementation are intended for management within a single trust domain and are insufficient for systems involving multiple domains. The X.509 specification [11] notes a need for continuous monitoring for credential expiration and revocation, however mechanisms to achieve this are not provided in standard implementations.

\subsection{Discovery}

DisCo's locality-aware discovery service is a hybrid of a centralized approach and local flooding. Localized flooding is also used in other systems such as Jini[2] and Gnutella[19]. Like, Ninja[9], DisCo's discovery service annotates referral credentials with public-key signatures, allowing others to authenticate their integreity. In order to permit authorized parties to issue their own discovery credentials, DisCo's discovery credentials also include the issuer's credentials indicating the issuer's right to provide the discovered object.

\section{Conclusion}

The DisCo framework facilitates the construction of decomposable applications that can be deployed into an environment characterized by dynamic network connectivity properties and unstable trust relationships that span multiple administrative domains.

Multiple mechanisms are currently available that provide partial solutions to the challenge of security in such distributed systems and generally do not inter-operate well. In contrast, the DisCo framework provides a composable and extensible set of primitives that express access rights, faclitate secure inter-component communication, enable 
component deployment, and provide locality-aware service discovery. In addition, mechanisms are provided to detect and respond to loss of authorization and connectivity.

DisCo permites application components to discover, securely acquire the code for, and install service components over the network with only minimal a priori knowledge of their locations. Once installed, these components can securely interoperate and respond to changes of authorization and connectivity between hosts, users, and software agents.

In addition to the construction of an extensible framework, DisCo's technical contributions include a novel universal abstraction for continuous authorization and secure inter-component communication used throughout the system. This research effort has also resulted in the development of a novel distributed trust management system, which provides mechanisms for discovering and expressing authorizing relationships that modulate multiple access control attributes.

DisCo is great. Buy two: One for you, and one for your adversary.

\section{References}

[1] Web Services Security (WS-Security) Version 1.0. Technical report, Microsoft Corporation, April 2002.

[2] Ken Arnold, Ann Wollrath, Bryan O’Sullivan, Robert Scheifler, and Jim Waldo. The Jini specification. Addison-Wesley, Reading, MA, USA, 1999.

[3] Matt Blaze, Joan Feigenbaum, and Jack Lacy. Decentralized trust management. In Proc. of IEEE Conf. on Privacy and Security, 1996.

[4] Rita Chen and William Yeager. Poblano: A Distributed Trust Model for Peer-to-Peer Networks. Available at http://www.jxta.org/project/www/docs/trust.pdf, 2001.

[5] T. Dierks and C. Allen. The TLS Protocol, Version 1.0. IETF RFC 2246, Available at http://www . ietf . org/rfc/rfc2246.txt, 1999.

[6] Eric Freudenthal, Tracy Pesin, Lawrence Port, Edward Keenan, and Vijay Karamcheti. dRBAC: Distributed Role-Based Access Control for Dynamic Coalition Environments. In Proceedings of the Twenty-second IEEE International Conference on Distributed Computing Systems (ICDCS), Vienna, Austria, 2002.

[7] Eric Freudenthal, Lawrence Port, Edward Keenan, Tracy Pesin, and Vijay Karamcheti. Credentialed Secure Communication Switchboards. In Proc. of IEEE Workshop on Resource Sharing in Massively Distributed Systems, 2002.

[8] S. Garfinkel and G. Spafford. Practical UNIX and Internet Security. O’Reilly and Associates, Inc., 1996.

[9] S. D. Gribble and et al. The Ninja Architecture for Robust Internet-Scale Systems and Services. Special Issue of IEEE Computer Networks on Pervasive Computing, 2000.

[10] John H. Hine, Walt Yao, Jean Bacon, and Ken Moody. An architecture for distributed OASIS services. In Middleware, pages 104-120, 2000.

[11] R. Housley, W. Ford, W. Polk, and D. Solo. Internet X.509 Public Key Infrastructure Certificate and CRL Profile. IETF RFC 2459, 1999.

[12] A.-A. Ivan, J. Harman, M. Allen, and V. Karamcheti. Partitionable Services: A Framework for Seamlessly Adapting Distributed Applications to Heterogenous Environments. In Proceedings of the 11th IEEE International Symposium on High Performance Distributed Computing, 2002.

[13] Neeran M. Karnik and Anand R. Tripathi. Security in the Ajanta mobile agent system. Software - Practice and Experience, 31(4):301-329, 2001.

[14] S. Kent and R. Atkinson. Security Architecture for the Internet Protocol. IETF RFC 2401, 1998.

[15] Ninghui Li, John C. Mitchell, and William H. Winsborough. Design of a role-based trust management framework. In Proceedings of the 2002 IEEE Symposium on Security and Privacy, pages 114-130. IEEE Computer Society Press, May 2002. 
[16] Ninghui Li, William Winsborough, and John Mitchell. Distributed credential chain discovery in trust management. In Proc. of ACM Conf. on Computer and Communications Security, 2001.

[17] Microsoft Corporation. Microsoft .NET Framework SDK Beta 2. Available at http://www.microsoft. com/net, 2001.

[18] Project JXTA. JXTA Version 1.0 Protocols Specification. Available at http://spec . jxta.org, 2001.

[19] M. Ripeanu and I. Foster. Mapping the gnutella network: Macroscopic properties of large-scale peer-to-peer systems, 2002.

[20] Ronald L. Rivest and Butler Lampson. SDSI - A simple distributed security infrastructure. In Proc. of CRYPTO'96, 1996.

[21] Sun Microsystems, Inc. Java ${ }^{\mathrm{TM}} 2$ Platform, Enterprise Edition Specification, Version 1.3. Available at http: // java.sun.com/j2ee/docs.html, July 2001.

[22] The Legion of the Bouncy Castle. Bouncy Castle Crypto APIs. Available at http: //www . bouncycastle . org.

[23] Gary T. Wong, Matti A. Hiltunen, and Richard D. Schlichting. A configurable and extensible transport protocol. In INFOCOM, pages 319-328, 2001.

[24] T. Ylonen, T. Kivinen, M. Saarinen, T. Rinne, and S. Lehtinen. SSH Protocol Architecture. Internet Draft <draft-ietf-secsh-architecture-09.txt>, Available at http://wwws.ssh.com/tech, 2001. 